\title{
Ageing in the critical contact process: a Monte Carlo study
}

\author{
José J. Ramasco ${ }^{a}$, Malte Henkel ${ }^{b}$, Maria Augusta Santos ${ }^{a}$ and \\ Constantino A. da Silva Santos ${ }^{a}$ \\ ${ }^{a}$ Departamento de Física and Centro de Física do Porto, \\ Faculdade de Ciências, Universidade do Porto, \\ Rua do Campo Alegre 687, P-4169-007 Porto, Portugal. \\ ${ }^{b}$ Laboratoire de Physique des Matériauxł, Université Henri Poincaré Nancy I, \\ B.P. 239, F - 54506 Vandœuvre-lès-Nancy Cedex, France. \\ E-mail: jjramasc@fc.up.pt,henkel@lpm.u-nancy.fr, \\ mpsantos@fc.up.pt,cssantos@fc.up.pt
}

\begin{abstract}
The long-time dynamics of the critical contact process which is brought suddenly out of an uncorrelated initial state undergoes ageing in close analogy with quenched magnetic systems. In particular, we show through Monte Carlo simulations in one and two dimensions and through mean-field theory that time-translation invariance is broken and that dynamical scaling holds. We find that the autocorrelation and autoresponse exponents $\lambda_{\Gamma}$ and $\lambda_{R}$ are equal but, in contrast to systems relaxing to equilibrium, the ageing exponents $a$ and $b$ are distinct. A recent proposal to define a non-equilibrium temperature through the short-time limit of the fluctuation-dissipation ratio is therefore not applicable.

PACS numbers: 05.70.Ln, 64.60.Ht, 75.40.Gb
\end{abstract}

Submitted to: J. Phys. A: Math. Gen.

\section{Introduction}

It is a well-established fact, theoretically known for many years and also experimentally observed, that the long-time properties of the ageing behaviour in glassy systems can be rationally interpreted in terms of a scaling picture which allows to make sense of many peculiarities of these systems which were once thought to be non-reproducible, see [1]. It has since been understood that similar effects also occur in non-glassy, e.g. simple ferromagnetic systems. From a microscopic point of view, the basic mechanism appears to be the formation of correlated domains of a time-dependent typical linear size $\ell(t)$. For simple ferromagnets and other non-glassy systems, it is generally admitted that $\ell(t) \sim t^{1 / z}$, where $z$ is the dynamical exponent. Furthermore, if $\phi(t, \boldsymbol{r})$ stands for the 
order parameter of such a simple ferromagnet, it turned out that these non-equilibrium relaxation phenomena are most conveniently studied through two-time quantities such as the two-time connected autocorrelator $\Gamma(t, s)$ and the two-time linear autoresponse function $R(t, s)$ defined by

$$
\Gamma(t, s)=\langle\Delta \phi(t, \boldsymbol{r}) \Delta \phi(s, \boldsymbol{r})\rangle \quad, \quad R(t, s)=\left.\frac{\delta\langle\phi(t, \boldsymbol{r})\rangle}{\delta h(s, \boldsymbol{r})}\right|_{h=0}
$$

where $\Delta \phi(t, \boldsymbol{r})=\phi(t, \boldsymbol{r})-\langle\phi(t)\rangle$ are the time-dependent fluctuations of the order parameter and $h$ is the magnetic field conjugate to $\phi$. Causality implies that $R(t, s)=0$ for $t<s$. By definition, a system is said to undergo ageing, if $\Gamma(t, s)$ or $R(t, s)$ does not merely depend on the time difference $\tau=t-s$, but on both the observation time $t$ and the waiting time s. For recent reviews, see [2, 3, 4, 15, 6, 17.

From experimental, numerical and analytical studies on ageing glasses and ferromagnets it has been learned that these systems may display dynamical scaling in the long-time limit [1, 2, 3, 4, 5, 6, 17. Specifically, consider the two-time functions in the ageing regime $t \gg t_{\text {micro }}, s \gg t_{\text {micro }}$ and $\tau=t-s \gg t_{\text {micro }}$, where $t_{\text {micro }}$ is some microscopic time. Then one expects the scaling behaviour

$$
\Gamma(t, s) \sim s^{-b} f_{\Gamma}(t / s), R(t, s) \sim s^{-1-a} f_{R}(t / s)
$$

where the scaling functions $f_{\Gamma, R}(y)$ have the following asymptotic behaviour for $y \rightarrow \infty$

$$
f_{\Gamma}(y) \sim y^{-\lambda_{\Gamma} / z}, f_{R}(y) \sim y^{-\lambda_{R} / z} .
$$

Here $\lambda_{\Gamma}$ and $\lambda_{R}$ are called the autocorrelation [8, 9] and autoresponse [10] exponents, respectively.

Up to present, most systems studied were such that they relax towards an equilibrium steady-state. Here we wish to ask what aspects of the ageing phenomenology remain if time-dependent systems with a non-equilibrium steady-state are considered. Besides the obvious question concerning an eventual scaling behaviour of two-time observables, we shall be specifically interested in the following two points.

(i) For systems with detailed balance, it is convenient to measure the distance of a system from equilibrium through the fluctuation-dissipation ratio [1]

$$
X(t, s):=T R(t, s)\left(\frac{\partial \Gamma(t, s)}{\partial s}\right)^{-1} .
$$

At equilibrium, the fluctuation-dissipation theorem (FDT) states that $X(t, s)=1$. Systems with an equilibrium steady-state may also be characterized through the limit fluctuation-dissipation ratio

$$
X_{\infty}=\lim _{s \rightarrow \infty}\left(\lim _{t \rightarrow \infty} X(t, s)\right) .
$$

If the ageing occurs at the critical temperature $T_{c}, X_{\infty}$ should be an universal number [12, 13, 3] and this has been confirmed in a large variety of systems in one and two space dimensions [13, 14, 15, 16, 17, whereas the value $X_{\infty}=0$ is expected for temperatures $T<T_{c}$. The order of the limits in (15) is important, since 
$\lim _{t \rightarrow \infty}\left(\lim _{s \rightarrow \infty} X(t, s)\right)=1$ always. On the other hand, Sastre et al. [16] asserted recently that a genuinely non-equilibrium temperature might be defined through

$$
\frac{1}{T_{\mathrm{dyn}}}:=\lim _{s \rightarrow \infty}\left(\lim _{t-s \rightarrow 0} R(t, s)\left(\frac{\partial \Gamma(t, s)}{\partial s}\right)^{-1}\right)
$$

and confirmed this through explicit calculation in the $2 D$ voter model as well as in other connected spin systems that do not satisfy the detailed balance condition.

Does their definition (6) extend to more general non-equilibrium systems (in particular those exhibiting an absorbing phase)?

(ii) For ageing simple magnets, it has been proposed that dynamical scaling might be generalized to the larger local scale-invariance [18. Such local scaletransformations, with dilatation factors depending locally on space and on time, may indeed be constructed for any given value of $z$. From the condition that $R(t, s)$ transforms covariantly under the action of local scale transformations it follows [18, 19]

$$
R(t, s)=r_{0}\left(\frac{t}{s}\right)^{1+a-\lambda_{R} / z}(t-s)^{-1-a},
$$

where $r_{0}$ is a normalization constant. This prediction has been confirmed in several models, notably the kinetic Ising model with Glauber dynamics, both in the bulk [19, 20] and close to a free surface [21, the kinetic XY model with a non-conserved order parameter [22, 23], for the Hilhorst-van Leeuwen model [21] and several variants of the exactly solvable spherical model [19, 13, 24, 23. .§ Very recently, the autocorrelation $\Gamma(t, s)$ could be predicted [25] from local scale-invariance for phase-ordering, where $z=2$. This prediction has been confirmed in the GlauberIsing, kinetic spherical and critical voter models and also for the free random walk [23, 25]. For recent reviews, see [26, 6].

Is there further evidence in favour of local scale-invariance in statistical systems without detailed balance?

Probably the simplest kinetic system far from equilibrium and without detailed balance is the celebrated contact process which has a steady-state transition in the directed percolation universality class. We shall therefore use this model in order to gain insight into the rôle of conditions such as detailed balance into the phenomenology of ageing behaviour. A complementary paper studies the same model through the density-matrix renormalization group [27]. In section 2, we recall the definition of the model. In section 3 , we discuss the computation of correlators either for the non-critical system or at the critical point. In section 4 , we define the response function and estimate it at criticality. After that, in section 5, we discuss the possibility of a generalization of the FDT for this model and in particular whether a non-equilibrium temperature may be defined through eq. (6). Finally, we conclude in section 6. In the appendix, the mean-field theory of ageing in the contact process is discussed.

$\S$ A technical complication arises for the $1 D$ Glauber-Ising model where the construction of the Liealgebra generators of local scale-invariance must be generalized, see appendix $\mathrm{C}$ in [23]. 


\section{The model}

The contact process (CP) was originally conceived as a simple model to describe epidemic disease propagation [28, 29]. Since then, CP has become a paradigm of Directed Percolation (DP), arguably the most common universality class of nonequilibrium phase transitions with an absorbing state. It is precisely the existence of an absorbing state that ensures that this model does not satisfy the detailed balance condition. This property stimulates our interest to try and characterize the model's dynamics in a new way.

We begin by recalling the definition of the model. In the contact process, the states of the system are described by a discrete variable, $n_{i}(t)$, defined on the sites $i$ of a hypercubic lattice. The possible values of $n_{i}$ are 1 or 0 depending on whether the site $i$ is occupied or empty. The dynamics is defined as follows: for each time step, a site $i$ of the lattice is randomly selected. If $i$ is occupied, that particle vanishes with probability $p$. Otherwise, with probability $1-p$, a new particle is created on one of the nearest neighbours of $i$ chosen at random (if that new site was still empty). When the control parameter $p$ is varied, the model exhibits a continuous phase transition from an active phase, where the mean density $\langle n(t)\rangle$ tends to a constant value $\bar{n}$ in the stationary state, to an absorbing phase, with zero final density. Separating these two phases, there is a critical point located at $p_{c}=0.2326746(5)$ in $1 D$ [30, 31] (the number in brackets gives the uncertainty in the last digit), and at $p_{c}=0.37753(1)$ in $2 D$ [31. We have arbitrarily fixed the initial condition of our system such that the particles are randomly distributed throughout the lattice with a mean initial density of $n_{0}=\langle n(0)\rangle=0.8$. The linear system sizes used are $L=10^{4}$ in $1 D$ and $L=300$ in $2 D$, respectively; the typical number of disorder realizations considered in simulations goes up to $2 \times 10^{4}$ in one dimension and $7 \times 10^{3}$ in $2 D$.

The introduction of an external field in the model will be necessary to estimate a response function. For this model, the external field $h$ corresponds to an additional probability of creation or destruction of particles, depending on the sign of $h$.

\section{Two-time autocorrelation functions}

A crucial difference between the CP and usual spin models is that starting from a zerodensity initial state (corresponding to zero magnetization in magnets) is not possible. This configuration corresponds to the absorbing state of the CP and once the system falls there, no further evolution occurs. Therefore, the spatially averaged mean density $\langle n(t)\rangle$ is positive during the time the systems evolves. For example, it decays as a power law, $\langle n(t)\rangle \sim t^{-\delta}$, at the critical point $p=p_{c}$. This fact implies that the two-time autocorrelation function defined as

$$
C(t, s)=\langle n(t, \boldsymbol{r}) n(s, \boldsymbol{r})\rangle,
$$



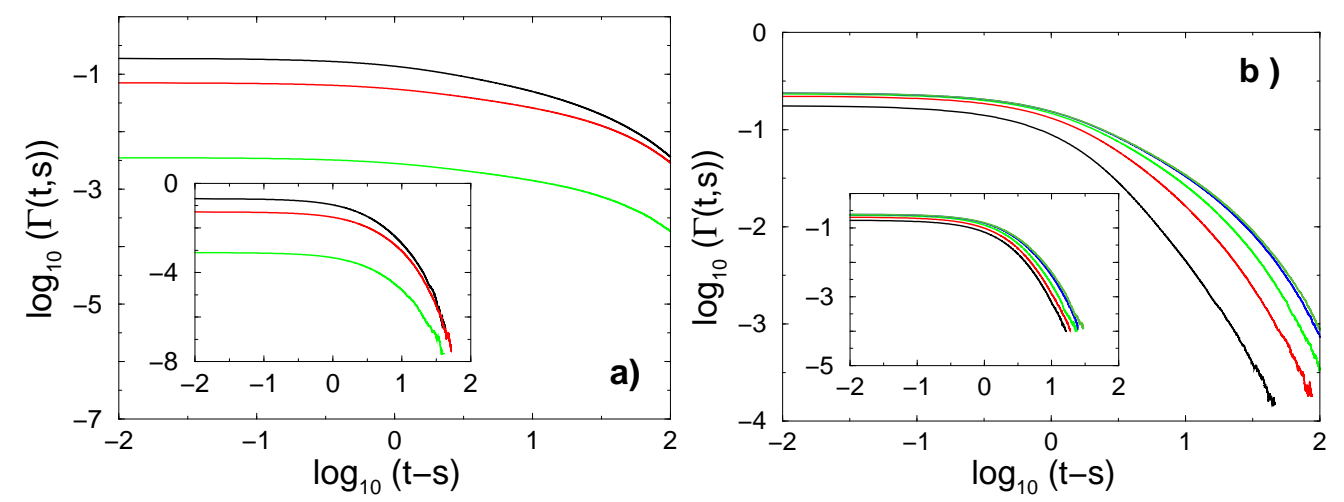

Figure 1. Connected autocorrelation function $\Gamma(t, s)$ for the $\mathrm{CP}$ for several values of the waiting time $s$. In a) the steady-state is in the absorbing phase. The main plot shows the $1 D$ system with $p=0.286$ and the inset shows the $2 D$ system with $p=0.56$. In b) the steady-state is in the active phase with $p=0.2$ in the main plot for $1 D$ and $p=0.286$ in the inset for $2 D$. The system sizes are $L=10^{4}$ in $1 D$ and $L=300$ in two dimensions. The waiting times in a) are from bottom to top $s=[30,100,300]$ and $s=[3,10,30]$ in the inset. In b), they are, from bottom to top, $s=[1,10,30,100,1000]$ and $s=[0.1,1,3,10,100]$ in the inset.

which is widely used in spin systems or glassy models, is no longer equal to the connected autocorrelation function or covariance that reads

$$
\Gamma(t, s)=\langle\Delta n(t, \boldsymbol{r}) \Delta n(s, \boldsymbol{r})\rangle,
$$

where $\Delta n(t, \boldsymbol{r})=n(t, \boldsymbol{r})-\langle n(t)\rangle$. Both functions are related by means of the expression

$$
\Gamma(t, s)=C(t, s)-\langle n(t)\rangle\langle n(s)\rangle .
$$

In eq. (10), the second term on the right behaves asymptotically as

$$
\langle n(t)\rangle\langle n(s)\rangle \sim \begin{cases}(t s)^{-\delta} & ; \text { if } p=p_{c} \\ \bar{n}^{2} & ; \text { if } p<p_{c}\end{cases}
$$

Therefore, for $p \leq p_{c}$ it follows that $C(t, s)$ and $\Gamma(t, s)$ do not share the same asymptotic behaviour.

Once the correlations have been defined, we are in position to deal with a first physical aspect of the temporal evolution of the contact process. It is well-known that away from the critical point, the model has a finite relaxation time towards its steadystate. That is, the mean density decays exponentially fast to its stationary value $\bar{n}$ : zero in the absorbing phase or a positive quantity in the active one, see [28]. In Figure 1], the transient behaviour of $\Gamma(t, s)$ for $t-s$ relatively small is shown for a system evolving to the steady-state in the absorbing phase. We see that, not only the density, but also its fluctuations with respect to $\langle n\rangle$ are vanishing, as described by the drop of the $\Gamma$-curves. The signature of a finite relaxation time may be noted in the persistence of the curve shape throughout the system evolution. || Next, we consider whether a similar behaviour \| Far above $p_{c}$, one has for large $s C(t, s) \sim \Gamma(t, s) \sim\langle n(t)\rangle$, see [27] for details. 


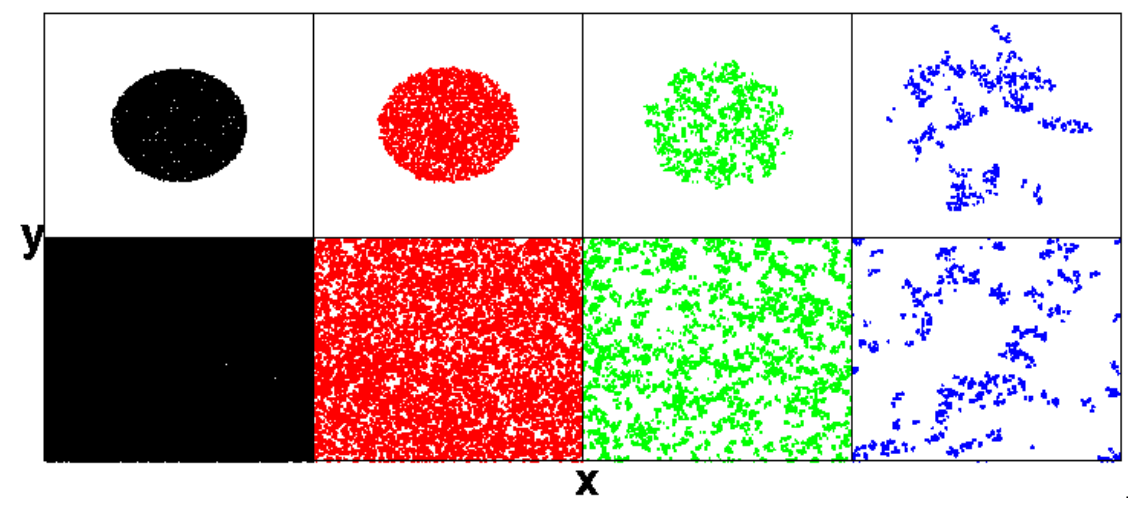

Figure 2. Microscopic evolution of clusters in the critical $2 D$ contact process. The lattice size is, in both series, $1000 \times 1000$. The initial condition of the upper plots is a full circle with radius 100 placed in the center of the lattice, while below it is a full lattice. The times are $t=[2,20,200,2000]$ for the upper figures and $t=[20,200,2000,20000]$ for the bottom snapshots.

can be seen in the active phase as well. In Figure 1b, the connected correlation function is displayed for a system in the active phase in both $1 D$ and $2 D$. The curves move upward during a first period of waiting times before they collapse, which implies that the TTI condition is fulfilled in this phase.

We point out that the observation of TTI at large enough waiting times in the active phase of the contact process is qualitatively different from what is found in ferromagnets quenched into their ordered phase. This may be understood qualitatively from the Ginzburg-Landau functional by observing that for ferromagnets there are two stable competing steady-states while for the contact process there is only a single one, to which the system relaxes rapidly.

From now on, we concentrate on the behaviour at criticality. In spin systems with a global symmetry (e.g. an up-down symmetry in the Ising model), the critical dynamics is based on the growth of spin domains whose average size, associated to the correlation length, increases as a power law in time, $\ell(t) \sim t^{1 / z}$ where $z$ is the dynamical exponent. The time-evolution of typical configurations of the critical CP in two dimensions is shown in Figure 2. Note the substantial difference with respect to bulk ferromagnetic spin systems: no domain walls are formed and, at the same time, the number of occupied sites decreases in time. This kind of ageing behaviour, not driven by a surface tension, has already been described in the $2 D$ voter model universality class [37]. Recently, it was pointed out that cluster dilution may also occur in the early stages of surface ageing of magnetic systems 38. As we shall see, the asymptotic dilution of clusters leads to new features in the scaling description of the ageing process of the CP.

In analogy with the behaviour of magnetic systems at the critical point, a scaling ansatz of the type 


$$
\begin{array}{ll}
\Gamma(t, s)=s^{-b} f_{\Gamma}(t / s), & f_{\Gamma}(y) \sim y^{-\lambda_{\Gamma} / z} \text { if } y \rightarrow \infty \\
C(t, s)=s^{-b} f_{C}(t / s), & f_{C}(y) \sim y^{-\lambda_{C} / z} \text { if } y \rightarrow \infty
\end{array}
$$

may be expected for the correlation functions, where the exponents $b, \lambda_{C} / z$ and $\lambda_{\Gamma} / z$ are to be determined. In Figure 3 , the critical evolution of $\Gamma(t, s)$ is plotted for several values of the waiting time $s$, over against $t-s$. Time-translation invariance is now clearly broken, and we find ageing effects for both one- and two-dimensional systems. The data can be collapsed when plotted over against $t / s$, as shown in Figure 3b which provides evidence for the validity of the scaling relation eq. (12). The values of the exponent $b$ used for the collapse were obtained from the scaling relation [35, 36]

$$
b=\frac{2 \beta}{\nu_{\|}}=\frac{(d-2+\eta)}{z}=2 \delta,
$$

which remains valid for the DP universality class. Here, $\beta$ is the static exponent which describes the variation of the order parameter close to criticality $\bar{n} \sim\left(p_{c}-p\right)^{\beta}$, $\nu_{\|}$is the temporal correlation-length exponent, $\eta$ is the static exponent of the order parameter spatial correlations, $\delta$ is the exponent controlling the critical decrease of the mean density and $z$ is the dynamic exponent (their values are summarized in table 1 ).

The exponent $\lambda_{\Gamma} / z$ may be estimated from the collapse of Figure 3b, with reasonable numerical accuracy. We find $\lambda_{\Gamma} / z=1.9 \pm 0.1$ in $1 D$ and $\lambda_{\Gamma} / z=2.8 \pm 0.3$ in $2 D$. If the critical $\mathrm{CP}$ were a Markov process, these exponents might be calculated from the global persistence exponent $\theta_{g}$ by means of the scaling relation [39, 40, 41]

$$
\frac{\lambda_{\Gamma}}{z}=\theta_{g}-\frac{2(1-d)-\eta}{2 z},
$$

This expression predicts $\lambda_{\Gamma} / z=1.98(2)$ in $1 D$ and $\lambda_{\Gamma} / z=3.5(5)$ in $2 D$, not too far from our numerical measurements. Still, the deviations from the directly measured
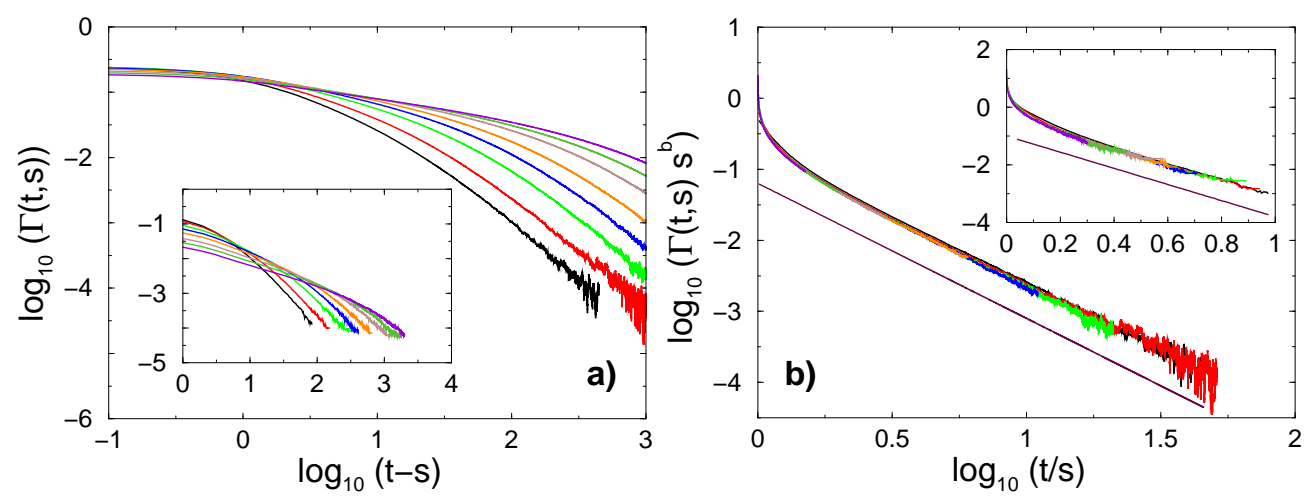

Figure 3. a) Evolution of the connected autocorrelation function at criticality. The curves in the inset are for two dimensions and the main plots for $1 D$. The waiting times are in both cases $s=[10,20,50,100,200,500,1000,2000]$ from left to right. In b) the collapse of the previous curves using Eqs. (12) 131) is shown. The straight lines yield exponent values of $\lambda_{\Gamma} / z=1.9$ in $1 D$ and of $\lambda_{\Gamma} / z=2.8$ in $2 D$. 

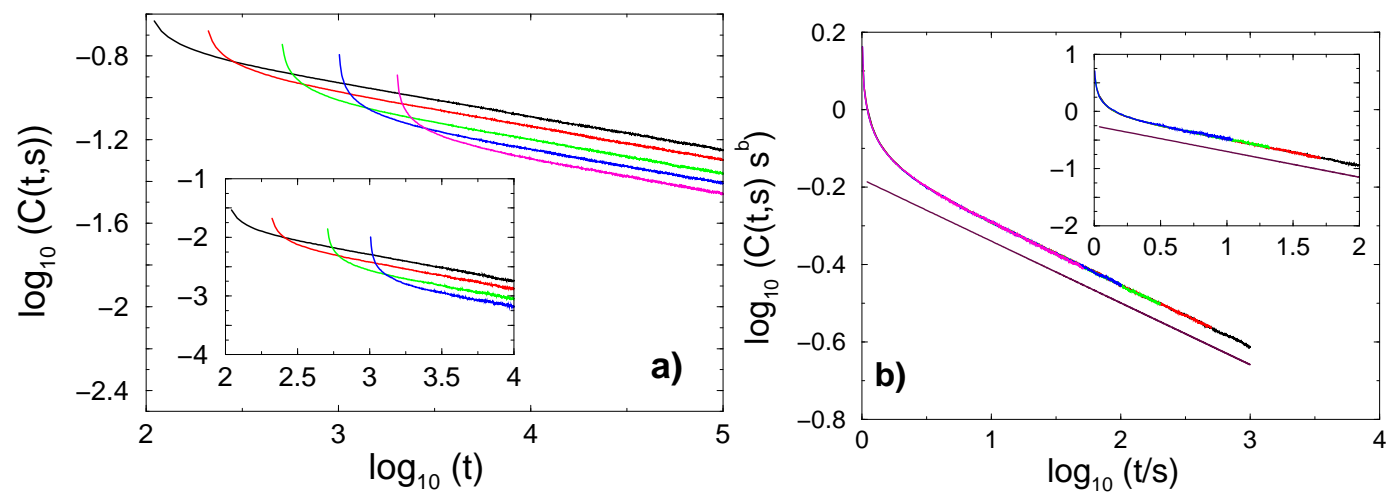

Figure 4. a) Two-time autocorrelation function $C(t, s)$ for the contact process in $1 D$ (main plots) and in $2 D$ (insets). The waiting times are from top to bottom $s=[100,200,500,1000]$ in both cases; for one dimension the value $s=2000$ is also included. These curves are collapsed in b) employing the scaling ansatz of Eq. [12] 13]. The straight lines have a slope $-\delta$.

values appear to be significant, pointing towards the possible existence of temporal long-range correlations and, hence, of an effective non-markovian dynamics.

The evolution of $C(t, s)$ is shown in Figure 4 for a range of waiting times. The collapse of these curves with the scaling ansatz of Eq. (12) is also shown. From this collapse, we make the identification $\lambda_{C} / z=\delta$ and also confirm the relation $b=2 \delta$. As expected, we have found a different asymptotic behaviour for $\Gamma(t, s)$ and $C(t, s)$. These results in $1 D$ and $2 D$ can be compared with the mean-field prediction in the scaling limit $s \rightarrow \infty$ with $y=t / s$ kept fixed (see the appendix)

$$
C(t, s) \sim \frac{1}{t s}=s^{-2}\left(\frac{t}{s}\right)^{-1} .
$$

This is in perfect agreement with the scaling ansatz eq. (12b) since the mean-field value for the exponent $\delta$ is $\delta_{\mathrm{MF}}=1$ (see table 1 ).

\section{The two-time autoresponse function}

The linear autoresponse function at time $t$ to an external perturbation by the field $h$ at time $s$ is defined as

$$
R(t, s)=\left.\frac{\delta\langle n(t)\rangle}{\delta h(s)}\right|_{h=0} .
$$

Unfortunately, this function is very difficult to estimate directly. Hence, as a general rule, the susceptibility is measured instead. It is common to work with the zero-fieldcooled (ZFC) susceptibility (we keep the terminology of magnetic spin systems by abuse of language)

$$
\chi_{\mathrm{ZFC}}(t, s)=\chi(t, s)=\int_{s}^{t} \mathrm{~d} u R(t, u) .
$$




\begin{tabular}{clllc}
\hline Exponent & $d=1$ & $d=2$ & mean-field & Reference \\
\hline$\beta$ & $0.27649(4)$ & $0.583(4)$ & 1 & {$[28]$} \\
$\nu_{\|}$ & $1.73383(3)$ & $1.295(6)$ & 1 & {$[28]$} \\
$z$ & $1.58074(4)$ & $1.766(2)$ & 2 & {$[28]$} \\
$\delta$ & $0.15947(3)$ & $0.4505(10)$ & 1 & {$[28]$} \\
$\eta$ & $1.50416(7)$ & $1.59(1)$ & 2 & {$[28]$} \\
$\theta_{g}$ & $1.50(2)$ & $2.5(5)$ & - & {$[39$, 40, 41] } \\
\hline$b$ & $0.31894(6)$ & $0.901(2)$ & 2 & \\
$\lambda_{\Gamma} / z$ & $1.9(1)$ & $2.8(3)$ & - & \\
$a$ & $-0.57(10)$ & $0.3(1)$ & $\frac{d}{2}-1$ & \\
$\lambda_{R} / z$ & $1.9(1)$ & $2.75(10)$ & $\frac{d}{2}+2$ & \\
\hline
\end{tabular}

Table 1. Exponents for the directed percolation universality class in one and two dimensions and in mean-field theory. The exponent $b=2 \delta$ and furthermore $\lambda_{C} / z=\delta$. The numbers in brackets give the estimated uncertainty in the last digit(s).

To measure this, one may consider the following dynamical rules. First, the system evolves according to the original CP rules, until the waiting time $s$ has elapsed. After that, two copies of the systems, A and B, are kept evolving in parallel. The copy A continues its evolution without perturbation, while copy B is subjected to an external field $h$. This field, as explained in section 2, represents a certain probability of creation $(h>0)$ or destruction $(h<0)$ of a particle whenever the selected site is empty or full, respectively. The susceptibility is then calculated as the limit $h \rightarrow 0$ of the ratio between the difference of the densities of the two copies and $h$ :

$$
\chi(t, s)=\lim _{h \rightarrow 0} \frac{\left\langle n_{B}(t)-n_{A}(t)\right\rangle}{|h|},
$$

where $h$ is turned on at time $s$ and kept until time $t$. However, the application of this method to the contact process is not straightforward, since a field $h \neq 0$ suppresses the phase-transition and hence the ageing behaviour. For spin systems, this difficulty may be side-stepped by using a spatially random magnetic field $h_{i}= \pm h[32$. Although this method may be applied to CP, the random field still brings the system out of its critical point, since there is no symmetry between the two states of the variable $n_{i}$. Consequently, to measure the susceptibility, there is no option but to force the system out of criticality. We have checked that applying an uniform field to the whole system is numerically more efficient than utilizing a random one. Therefore we shall employ the former method in our calculations. We stress that, in both procedures, a small enough value of $h$ must be taken to prevent saturation for the times considered in the simulations. From studies of the magnetization-reversal transition in spin systems it is known that ageing behaviour can be found if $h$ is small enough [33. In Figure 5a, the susceptibility is depicted for a fixed waiting time and several values of the external field. The asymptotic behaviour $(h \rightarrow 0)$, which corresponds to the early $\tau=t-s$ regime, may be there clearly observed. In addition, in Fig. 5 b, the same magnitude 

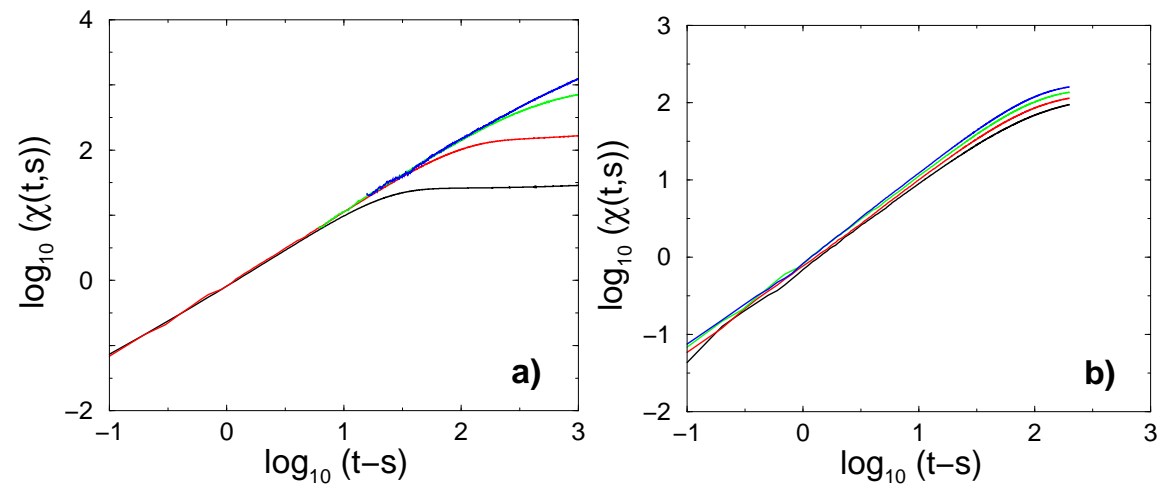

Figure 5. a) The ZFC susceptibility $\chi(t, s)$ as a function of $t-s$ for the onedimensional contact process at $p=p_{c}$. The external field is changed for the different curves, from bottom to top $h=\left[10^{-2}, 10^{-3}, 10^{-4}, 10^{-5}\right]$, while the waiting time is maintained at $s=1000$. In b), the external field is fixed at $h=10^{-3}$ and $\chi(t, s)$ is represented for several waiting times, from bottom to top, $s=[250,500,1000,2000]$.

is represented for a range of waiting times. This shows that there is a time window available for studies of the ageing behaviour of the linear response.

The interpretation of the zero-field-cooled susceptibility in terms of the scaling behaviour of the linear autoresponse function $R(t, s)$ meets with a further difficulty. Naïvely, one would simply insert the assumed scaling form eq. (21) into (17). This simplistic procedure would lead to

$$
\chi(t, s) \stackrel{?}{=} \int_{s}^{t} \mathrm{~d} u u^{-1-a} f_{R}(t / u)=t^{-a} \int_{1}^{t / s} \mathrm{~d} w w^{a-1} f_{R}(w)=s^{-a} f_{M}(t / s)
$$

However, as pointed out in [34, in doing so one neglects the important condition $t-s \gg t_{\text {micro }}$ necessary for the validity of eq. (22). Indeed, it can be shown that taking this condition into account rather leads to 34

$$
\chi(t, s)=\chi_{0}+s^{-A} g_{M}(t / s)+\mathrm{O}\left(s^{-a}\right)
$$

such that the exponent $A \geq 0$ is in general unrelated to $a$ and where $\chi_{0}$ is some constant. For example, for phase-ordering spin systems which are at a temperature above their roughening temperature $T_{R}$, one always has $A<a$ 34. Hence the term of order $\mathrm{O}\left(s^{-a}\right)$ in (201), coming from the integral (19) over the autoresponse function, merely furnishes a finite-time correction (see the appendix for a related difficulty of using $\chi_{\mathrm{ZFC}}$ which arises in mean-field theory).

These difficulties may be circumvented by using the integrated response in a setup similar to a Thermoremanent Magnetization (TRM) experiment, with a small modification, close in spirit to the 'intermediate' protocol proposed in 34. To do so, we start with a system evolving with the usual contact process rules. After a time $s-\tau_{c}$, the system is split in two copies, A and B. A continues its evolution without perturbation, whereas B is subjected to an external field $h$. The field is switched off at 
time $s$, and from then on we track the difference between the two copies, which is called the thermoremanent density:

$$
\rho(t, s)=\int_{s-\tau_{c}}^{s} \mathrm{~d} u R(t, u)=\lim _{h \rightarrow 0} \frac{\left\langle n_{B}(t)-n_{A}(t)\right\rangle}{|h|}
$$

where $h$ is now turned on at time $s-\tau_{c}$ and switched off at time $s$. In addition, $\tau_{c}$ must satisfy the relation $\tau_{c} \leq s-$ actually, this scheme would correspond to a standard TRM experiment if $\tau_{c}=s$. In addition, $\tau_{c}$ must also be much smaller than the relaxation time of copy B to avoid that $\rho(t, s)$ becomes a function of the difference of times $t-s$ alone 42. The scaling of $\rho$ may be obtained from inserting eq. (17) of local scale-invariance into (21). It can be shown 34 that the leading corrections to scaling are of order $\mathrm{O}\left(s^{-\lambda_{R} / z}\right)$ which is negligible at criticality.

A last point has to be discussed before the scaling of the integrated response $\rho$ as calculated here can be analysed quantitatively. Consider the linear space-time response function

$$
R=R\left(t, s ; \boldsymbol{r}-\boldsymbol{r}^{\prime}\right):=\left.\frac{\delta\langle n(t, \boldsymbol{r})\rangle}{\delta h\left(s, \boldsymbol{r}^{\prime}\right)}\right|_{h=0}
$$

In contrast to the situation usually considered in magnetism, where the field $h$ is modelled by spatially uncorrelated random variables, we use here an uniform magnetic field with spatial correlation, as explained before. This implies that the measured TRM becomes for $s \gg \tau_{c}$

$$
\rho(t, s)=\frac{1}{L^{2 d}} \sum_{\boldsymbol{r}} \sum_{\boldsymbol{r}^{\prime}} R\left(t, s ; \boldsymbol{r}-\boldsymbol{r}^{\prime}\right)=\widehat{R}_{\mathbf{0}}(t, s),
$$

where the sums runs over the entire lattice of linear size $L$ and $\widehat{R}_{\boldsymbol{k}}(t, s)$ is the Fourier transform of $R(t, s ; \boldsymbol{r})$ at momentum $\boldsymbol{k}$. In order to understand the scaling behaviour of $\widehat{R}_{\mathbf{0}}(t, s)$, we recall that local scale-invariance predicts for any given value of $z$ the following form of the space-time response [18]

$$
R(t, s ; \boldsymbol{r})=R(t, s) \Phi(|\boldsymbol{r}| / \xi),
$$

where $R(t, s)$ is the autoresponse function, $\xi \sim(t-s)^{1 / z}$ and $\Phi$ is a scaling function which can be obtained from a linear differential equation of fractional order. $\uparrow$ Therefore

$$
\widehat{R}_{\mathbf{0}}(t, s)=\int_{\mathbb{R}^{d}} \mathrm{~d} \boldsymbol{r} R(t, s ; \boldsymbol{r}) \sim R(t, s) \xi^{d} \sim R(t, s)(t-s)^{d / z},
$$

where we assumed that the scaling function $\Phi(u)$ falls off sufficiently rapidly for $u$ large such that the Fourier transform exists. If the autoresponse function scales asymptotically (i.e. for $t / s \gg 1$ ) as

$$
R(t, s) \sim s^{-1-a}\left(\frac{t}{s}\right)^{-\lambda_{R} / z}
$$

we expect for the TRM the scaling behaviour

$$
\rho(t, s) \sim \hat{R}_{0}(t, s) \sim s^{-1-a+d / z}\left(\frac{t}{s}\right)^{\left(d-\lambda_{R}\right) / z}
$$



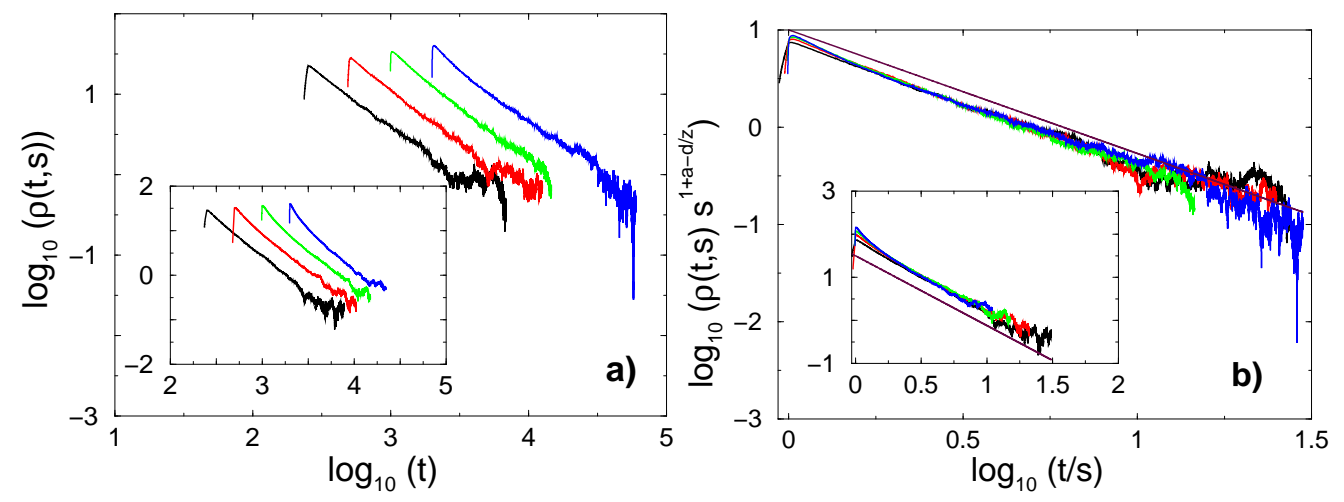

Figure 6. a) Temporal evolution of the 'thermoremanent' density $\rho(t, s)$ (see eq. (21)) for several waiting times, from bottom to top $s=[250,500,1000,2000]$. The main plots are for one dimensional systems and the insets for $2 D$. In b), we collapse the curves of a) according to dynamical scaling eq. (27). The slopes of the straight lines are -1.27 in $1 D$ and -1.62 in $2 D$.

In Figure $6 \mathrm{a}$, the evolution of $\rho$ is plotted for several values of the waiting time. The strength of the field used in the simulation is $h=10^{-3}$ and $\tau_{c}=25$. The ageing behaviour is clearly visible. Replotting the data according to the dynamical scaling behaviour expected from eq. (27) (figure [6), we find a nice data collapse for the exponent values

$$
1+a-\frac{d}{z}=\left\{\begin{aligned}
-0.20(10) & ; \text { in } 1 D \\
0.17(10) & ; \text { in } 2 D
\end{aligned}\right.
$$

Taking the slopes of the curves in figure $6 \mathrm{~b}$ we get

$$
\frac{\lambda_{R}-d}{z}= \begin{cases}1.27(10) & ; \text { in } 1 D \\ 1.62(10) & ; \text { in } 2 D\end{cases}
$$

from which the exponent values of $a$ and $\lambda_{R}$ given in table 1 are obtained.

It is satisfying that for $d=1$, the results of a complementary DMRG calculation 27] are consistent with ours. Our results may also be compared with the prediction of mean-field theory, as derived in the appendix

$$
R(t, s) \sim\left(\frac{t}{s}\right)^{-2}(t-s)^{-d / 2}
$$

The mean-field values for the exponents are then $a=\frac{d}{2}-1$ and $\lambda_{R} / z=\frac{d}{2}+2$. They should become exact for $d>4$ and are not too far from the simulational results in lower dimensions.

Comparing with the exponents obtained from the autocorrelation function in section 3 , we observe that, within numerical errors, the autocorrelation and autoresponse exponents agree

$$
\lambda_{\Gamma}=\lambda_{R},
$$

I For $z=2$, it can be shown that $\Phi(u)=\exp \left(-\mathcal{M} u^{2}\right)$, where $\mathcal{M}$ is a dimensionful constant 18. 
but that the exponents $a$ and $b$ are different. In fact, within error bars they obey the following relation

$$
1+a=b=2 \delta .
$$

This last result leads to important consequences as we shall see in the next section. Finally, the uncertainty in the ageing exponents derived from our numerical data is too large for a direct check of the validity of local scale-invariance to be carried out. Nevertheless, our results can be related to the ones using a direct estimation of $R(t, s)$ via the DMRG [27] through the ansatz (24), which is based on local scale-invariance. The agreement between the two measurements provides indirect evidence for the existence of this symmetry in the CP. On the other hand, the prediction (77) of local scale-invariance is satisfied by our mean-field result (30) and in $1 D$, the validity of eq. (7) was directly confirmed from a DMRG calculation [27.

\section{Fluctuation-dissipation theorem}

Our observation that $a \neq b$ at criticality has important consequences. In particular, it follows that no analogue of the fluctuation-dissipation ratio can be defined in the critical contact process in a meaningful way. To see this, we use the scaling ansatz (12 ) for the connected correlation function and find

$$
\frac{\partial \Gamma(t, s)}{\partial s}=-s^{-b-1}\left[b f_{\Gamma}(t / s)+(t / s) f_{\Gamma}^{\prime}(t / s)\right] .
$$

where the prime denotes the derivative. This implies for a formally defined fluctuationdissipation ratio $R(t, s)(\partial \Gamma(t, s) / \partial s)^{-1}$ :

(i) a limit fluctuation-dissipation ratio, in analogy with (5), can only have a non-trivial value if simultaneously $a=b$ and $\lambda_{R}=\lambda_{\Gamma}$. From table 1 , we see that this is not the case for the critical contact process.

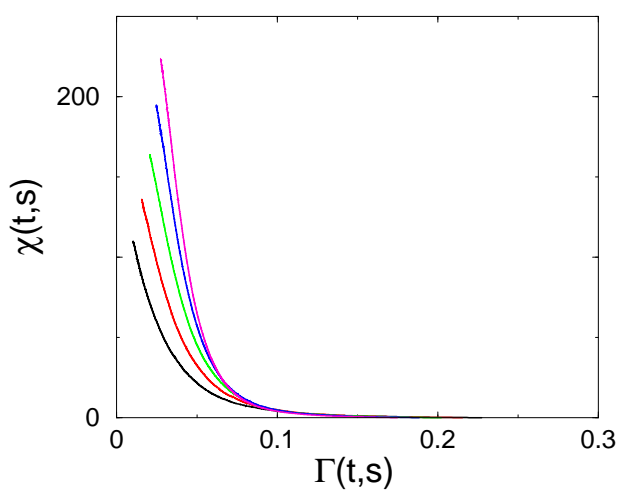

Figure 7. The ZFC susceptibility against the connected correlation function for a one-dimensional system and with different waiting times, from bottom to top $s=[250,500,1000,2000,5000]$. The external field was fixed at $h=7 \times 10^{-4}$. 
(ii) in contrast to the finding of Sastre et al. [16] for the critical voter model, there is no non-trivial limit $t-s \rightarrow 0$ inside the scaling regime of the fluctuation-dissipation ratio. Existence of such a limit requires $a=b$ as a necessary condition. From table 1 , that condition is not satisfied. Consequently, the supposedly generic definition of a non-equilibrium temperature -eq. (6]) - as proposed in [16] does not extend to the critical contact process and hence should reflect a peculiarity of the models with symmetric states. ${ }^{+}$

A more graphical way to arrive at the same conclusion is to plot the ZFC susceptibility $\chi(t, s)$ versus the connected correlation function $\Gamma(t, s)$ : if there were a generalized FDT, we should find a curve with a constant slope that corresponds to the inverse of the effective temperature. We do so in figure 7 for the $1 D$ case, which makes it clear that no such extension of the FDT exists, in contrast with results [16] of the $2 D$ critical voter model.

\section{Conclusions}

We have explored the rôle of the detailed balance condition in the slow non-equilibrium relaxation of a statistical system towards a steady-state. In order to do so, we have replaced a key property of physical ageing, namely that the basic microscopic processes are reversible, by a property encountered in chemical or biological ageing with their underlying irreversible microscopic processes. Consequently, the steady-state of the system can no longer be at equilibrium. One of our main questions was how the accepted scaling behaviour found in physical ageing might be modified in the absence of detailed balance and we tried to get some insight into this through a case study of the contact process, which is a paradigmatic example of systems with a steady-state phasetransition in the directed percolation universality class. We have studied the ageing of this model by analyzing two-time autocorrelation and response functions, obtained from Monte Carlo simulations and also analytically from the mean-field approximation. Our numerical results are in full agreement with those coming from an application of the density-matrix renormalization group to the same model [27].

Our results reemphasize the importance of having either at least two physically distinct and stable steady-states or else being at a continuous phase-transition in order to be able to observe the slow non-exponential relaxation towards a global steady-state which is associated with the phenomenological aspects of ageing behaviour, notably the breaking of time-translation invariance and of dynamical scaling. In this sense, it is natural that in the contact process ageing is only found if the steady-state is critical. We have shown that the phenomenological scaling description, previously developed for ageing magnetic systems, generalizes to the case at hand. In particular, we have

+ The basic of assumption of [16], namely that $X(t, s) \rightarrow 1$ in the short-time limit has been critically reexamined by Mayer and Sollich [43, who in the $1 D$ Glauber-Ising model at $T=0$ construct a defect-pair observable such that $\lim _{s \rightarrow \infty} X(s, s)=3 / 4$. 
presented evidence for the exponent relations

$$
\lambda_{\Gamma}=\lambda_{R}, \quad 1+a=b=2 \delta
$$

in one and two dimensions and in mean-field theory. While the first of those is generic in ageing magnets with an uncorrelated initial state, the second one is not and suggests that the non-equilibrium exponents $a$ and $b$ are in general distinct. ${ }^{*}$ It will be interesting to see whether or not the relations (34) hold at the critical points of other nonequilibrium universality classes. Eq. (34) implies that a recent attempt [16 to define a nonequilibrium temperature through an extension of the fluctuation-dissipation theorem cannot be generalized beyond the rather restricted context where this definition had been proposed.

\section{Acknowledgments}

We are grateful to T. Enss, M.A. Muñoz, A. Picone and U. Schollwöck for useful comments. This work was supported by the French-Portuguese integrated action under project F-14/04, and from the Portuguese Research Council (FCT) under grants SFRH/BPD/5557/2001 and SFRH/BPD/14394/2003.

\section{Appendix. Mean-field theory}

The contact process is described at the continuum level by the Reggeon Field Theory (RFT) [44. Let $\phi(t, \boldsymbol{r})$ represent the continuum order-parameter at the spatial position $\boldsymbol{r}$ and at time $t$. Taking into account the initial conditions, which is fundamental to analyze this problem, the RFT-action reads

$$
S=\int \mathrm{d} t \mathrm{~d} \boldsymbol{r}\left[\tilde{\phi}\left(\partial_{t} \phi-m \phi-D \nabla^{2} \phi\right)+g \phi^{2} \tilde{\phi}-g \phi \tilde{\phi}^{2}-n_{0} \delta(t) \tilde{\phi}\right],
$$

where $\tilde{\phi}$ is an auxiliary field, $m$ the mass term, $D$ the diffusion constant, $g$ a coupling constant and $n_{0}$ the initial density. The mean-field approximation amounts to treating (A1) as a classical action. This yields the following equations of motion

$$
\frac{\delta S}{\delta \tilde{\phi}(t)}=\left(\partial_{t}-m-D \nabla^{2}\right) \phi+g \phi^{2}-n_{0} \delta(t)=0
$$

and

$$
\frac{\delta S}{\delta \phi(t)}=\left(-\partial_{t}-m-D \nabla^{2}\right) \tilde{\phi}+2 g \phi \tilde{\phi}=0 .
$$

We can now evaluate the classical density and the classical response function. The term classical means here averaged with respect to action (A1) but only with the $\phi^{2} \tilde{\phi}$ vertex (diagrammatically, the term classical refers to the absence of loops in the diagrams). The classical density is given by the sum of all tree-diagrams which terminate with a

* For systems with detailed balance, the steady-state is an equilibrium state where the fluctuationdissipation theorem holds. This in turn implies $a=b$ [27. 
single propagator. If one evaluates these diagrams in momentum space, it follows that the $\delta(t) \tilde{\phi}(t)$ only make a contribution with $\boldsymbol{k}=0$ and therefore all diagrams at tree level have $\boldsymbol{k}=0$, see [45]. The classical density is found by Fourier-transforming eq. (A2). We find

$$
\hat{\phi}(\omega)=-\frac{n_{0}}{m+\mathrm{i} \omega}+\frac{g}{m+\mathrm{i} \omega} \int \frac{\mathrm{d} \omega^{\prime}}{2 \pi} \hat{\phi}\left(\omega-\omega^{\prime}\right) \hat{\phi}\left(\omega^{\prime}\right),
$$

where $\hat{\phi}(\omega)$ is the Fourier transform of $\phi(t)$. Note that the diffusion term has been disregarded since it gives no contribution to the final result, as explained above. Eq. (A4) can be iterated to obtain a perturbative series. For $m=0$, that is, at the critical point, the series can be summed yielding the exact solution

$$
\phi(t)=\frac{n_{0}}{1+g n_{0} t}
$$

Therefore, the classical two-time correlation function is given by

$$
C(t, s)=\phi(t) \phi(s)=\frac{n_{0}^{2}}{\left(1+g n_{0} s\right)\left(1+g n_{0} t\right)} .
$$

We can now turn to the computation of the response function. The Fourier transform of equation (A3) with respect to $\boldsymbol{r}$ reads

$$
-\partial_{t} \tilde{\phi}(\boldsymbol{k}, t)=m \tilde{\phi}(\boldsymbol{k}, t)-D \boldsymbol{k}^{2} \tilde{\phi}(\boldsymbol{k}, t)-2 g \phi(t) \tilde{\phi}(\boldsymbol{k}, t),
$$

where $\tilde{\phi}(\boldsymbol{k}, t)$ is the Fourier transform of $\tilde{\phi}(t, \boldsymbol{r})$. The above equation has the formal solution

$$
\tilde{\phi}(\boldsymbol{k}, t)=\tilde{\phi}(\boldsymbol{k}, s) e^{\left(-m+D \boldsymbol{k}^{2}\right)(t-s)} \exp \left[2 g \int_{s}^{t} \mathrm{~d} t^{\prime} \phi\left(t^{\prime}\right)\right] .
$$

At criticality, $m=0$ and using equation (A5) the above equation yields (setting $D=1$ )

$$
\tilde{\phi}(\boldsymbol{k}, t)=\tilde{\phi}(\boldsymbol{k}, s) e^{\boldsymbol{k}^{2}(t-s)}\left(\frac{1+g n_{0} t}{1+g n_{0} s}\right)^{2} .
$$

In order to calculate the response function is necessary to take $t>s$ and to multiply both sides of equation (A.9) by $\phi(-\boldsymbol{k}, t+\epsilon)$, where $\epsilon>0$. The fact that there is no inertial term in the action (A1) (that is, there are no second-order time derivatives) leads to the following boundary condition

$$
\lim _{\epsilon \rightarrow 0}\langle\phi(-\boldsymbol{k}, t+\epsilon) \tilde{\phi}(\boldsymbol{k}, t)\rangle=\lim _{\epsilon \rightarrow 0} \widehat{R}_{\boldsymbol{k}}(t+\epsilon, t)=1
$$

Consequently, the classical response function reads

$$
\langle\phi(-\boldsymbol{k}, t) \tilde{\phi}(\boldsymbol{k}, s)\rangle=\widehat{R}_{\boldsymbol{k}}(t, s)=e^{-\boldsymbol{k}^{2}(t-s)}\left(\frac{1+g n_{0} s}{1+g n_{0} t}\right)^{2}
$$

In real space, the space-time-dependent response function becomes

$$
R(t, s ; \boldsymbol{r})=\langle\phi(t, \boldsymbol{r}) \tilde{\phi}(s, \mathbf{0})\rangle=R(t, s) \exp \left(-\frac{\boldsymbol{r}^{2}}{4(t-s)}\right)
$$

where the autoresponse function is

$$
R(t, s)=\left(\frac{\pi}{t-s}\right)^{d / 2}\left(\frac{1+g n_{0} s}{1+g n_{0} t}\right)^{2}
$$


We are interested in these expressions in the ageing regime $s \gg 1, t-s \gg 1$. The the autocorrelation function reads

$$
C(t, s) \sim s^{-1} t^{-1}=s^{-2}\left(\frac{t}{s}\right)^{-1}
$$

and we read off the mean-field values $b_{\mathrm{MF}}=2$ and $\left(\lambda_{C} / z\right)_{\mathrm{MF}}=2$ of the exponents (mean-field theory being gaussian, $z_{\mathrm{MF}}=2$ is evident). The autoresponse function becomes

$$
R(t, s)=\pi^{d / 2}(t-s)^{-d / 2}\left(\frac{t}{s}\right)^{-2}
$$

in agreement with the form predicted by local scale-invariance and we have the exponent $a_{\mathrm{MF}}=\frac{d}{2}-1$ and $\left(\lambda_{R} / z\right)_{\mathrm{MF}}=\frac{d}{2}+2$. While simple mean-field does not yield a prediction for the connected correlation function $\Gamma(t, s)$, at least it furnishes expressions for the correlator and the linear response and their exponents which should become exact for $d>4$.

We finally illustrate the importance of taking both conditions $s \gg 1$ and $t-s \gg 1$, or equivalently $s \gg 1$ and $t / s \gg 1$, for the validity of the scaling form (2) into account. First, for the thermoremanent protocol (we take $\tau_{c}=s$ ) we find, using (A15)

$$
\rho_{\mathrm{TRM}}(t, s)=\int_{0}^{s} \mathrm{~d} u R(t, u) \sim t^{1-d / 2} \int_{0}^{s / t} \mathrm{~d} w w^{2}(1-w)^{-d / 2}
$$

which produces a well-defined scaling function for all values of $d$. On the other hand, for the zero-field-cooled protocol we have

$$
\chi_{\mathrm{ZFC}}(t, s)=\int_{s}^{t} \mathrm{~d} u R(t, u) \stackrel{?}{\sim} t^{1-d / 2} \int_{s / t}^{1} \mathrm{~d} w w^{2}(1-w)^{-d / 2}
$$

which diverges for all $d \geq 2$. This means that the scaling form (A15) cannot be used near the upper integration limit in (A17) and the contribution of the region around $w=1$ must be evaluated separately, without appealing to dynamical scaling (we remind the reader that the condition $t-s \gg 1$ is implicit in the continuum limit used to derive (A11).

After submission of this work, we became aware of a paper by Oerding and van Wijland [46] where the two-time connected autocorrelation function $\widehat{\Gamma}_{\mathbf{0}}(t, s)$ of reggeon field-theory is worked out in momentum space to one-loop order. They find a scaling behaviour quite analogous to the one observed here.

\section{References}

[1] L.C.E. Struik, Physical ageing in amorphous polymers and other materials, Elsevier (Amsterdam 1978).

[2] see the reviews by J.P. Bouchaud and by A.J. Bray in M.E. Cates and M.R. Evans (eds) Soft and fragile matter, IOP Press (Bristol 2000).

[3] C. Godrèche and J.-M. Luck, J. Phys. Cond. Matt. 14, 1589 (2002).

[4] L.F. Cugliandolo, in Slow Relaxation and non equilibrium dynamics in condensed matter, J-L Barrat, J Dalibard, J Kurchan, M V Feigel'man eds, Springer (Heidelberg 2003). 
[5] A. Crisanti and F. Ritort, J. Phys. A36, R181 (2003).

[6] M. Henkel, Adv. Solid State Phys. 44, 353 (2004).

[7] P. Calabrese and A. Gambassi, submitted to J. Phys. A.

[8] D.S. Fisher and D.A. Huse, Phys. Rev. B38, 373 (1988).

[9] D.A. Huse, Phys. Rev. B40, 304 (1989).

[10] A. Picone and M. Henkel, J. Phys. A35, 5575 (2002).

[11] L.F. Cugliandolo, J. Kurchan and G. Parisi, J. Physique I4, 1641 (1994).

[12] C. Godrèche and J.M. Luck, J. Phys. A33, 1151 (2000).

[13] C. Godrèche and J.M. Luck, J. Phys. A33, 9141 (2000).

[14] P. Calabrese and A. Gambassi, Phys. Rev. E66, 066101 (2002); E67, 036111 (2003); B66, 212407 (2002).

[15] M. Henkel and G.M. Schütz, J. Phys. A37, 591 (2004).

[16] F. Sastre, I. Dornic and H. Chaté, Phys. Rev. Lett. 91, 267205 (2003).

[17] C. Chatelain, J. Stat. Mech.: Theor. Exp. P06006 (2004).

[18] M. Henkel, Nucl. Phys. B641, 405 (2002).

[19] M. Henkel, M. Pleimling, C. Godrèche, and J.-M. Luck, Phys. Rev. Lett. 87, 265701 (2001).

[20] M. Henkel and M. Pleimling, Phys. Rev. E68, 065101(R) (2003).

[21] M. Pleimling, Phys. Rev. B70, 104401 (2004).

[22] S. Abriet and D. Karevski, Eur. Phys. J. B37, 47 (2004);

S. Abriet and D. Karevski, Eur. Phys. J. B41, 79 (2004).

[23] A. Picone and M. Henkel, Nucl. Phys. B688, 217 (2004).

[24] S.A. Cannas, D.A. Stariolo and F.A. Tamarit, Physica A294, 362 (2001).

[25] M. Henkel, A. Picone and M. Pleimling, Europhys. Lett. (2004) at press (cond-mat/0404464).

[26] M. Henkel, A. Picone, M. Pleimling and J. Unterberger, cond-mat/0307649

[27] T. Enss, M. Henkel, A. Picone and U. Schollwöck, J. Phys. A37 (2004) at press (cond-mat/0406147).

[28] H. Hinrichsen, Adv. Phys. 49, 815 (2000).

[29] G. Ódor, Rev. Mod. Phys. 76, 663 (2004).

[30] I. Jensen and R. Dickman, J. Stat. Phys. 71, 89 (1993).

[31] R. Dickman and J.K. Leal da Silva, Phys. Rev. E58, 4266 (1998).

[32] A. Barrat, Phys. Rev. E57, 3629 (1998).

[33] M. Paeßens and M. Henkel, J. Phys. A36, 8983 (2003).

[34] M. Henkel, M. Paeßens and M. Pleimling, Phys. Rev. E69, 056109 (2004).

[35] F. van Wijland, K. Oerding and H. Hilhorst, Physica A251, 179 (1998).

[36] M.A. Muñoz, R. Dickman, A. Vespignani and S. Zapperi, Phys. Rev. E59, 6175 (1999).

[37] I. Dornic, H. Chaté, J. Chave and H. Hinrichsen, Phys. Rev. Lett. 87, 045701 (2001).

[38] M. Pleimling and F. Iglói, Phys. Rev. Lett. 92, 145701 (2004).

[39] S.N. Majumdar, A.J. Bray, S.J. Cornell and C. Sire, Phys. Rev. Lett. 77, 3704 (1996).

[40] H. Hinrichsen and H.M. Koduvely, Eur. Phys. J. B5, 257 (1998).

[41] E.V. Albano and M.A. Muñoz, Phys. Rev. E63, 031104 (2001).

[42] M. Pleimling, Phys. Rev. E70, 018101 (2004).

[43] P. Mayer and P. Sollich, cond-mat/0405711.

[44] J.L. Cardy and R.L. Sugar, J. Phys. A13, L423 (1980).

[45] B.P. Lee, J. Phys. A27, 2633 (1994).

[46] K. Oerding and F. van Wijland, J. Phys. A31, 7011 (1998). 